resulted from such an evolutionary history. Finally, we recognize that evolution in Man's behaviour has become rapid relative to his lifetime, since mankind now gains new knowledge quickly and disseminates it efficiently, so that new situations for humanity can now develop in the space of years. This evolution is of a new kind, not concerned with Man's structure but with his ability to control and change his environment. It is still quite unclear where this new evolution may lead us.

This story is recounted in simple terms, with compelling logic. The original lectures were for a high-school audience, and this extended version is still suitable for them. But it has much interest for all, and I hope that it will become more widely known. It could perhaps be simplified more, or extended in several places where questions are left unanswered. The book will be valuable for scientists, since few are familiar with all of the areas covered. The presentation is stronger and more tightly argued in the earlier chapters concerned with chemistry and physics than in the chapters concerning Life, where the author's knowledge comes from other scientists. The story of Life is much more complicated than that of inorganic matter, and can only be outlined in such a short space.

Each reader will have his own short-list of significant topics not covered in this book. For example, the uniqueness of our situation on Earth might have been underlined by a brief comparison of Venus with Earth; why have two planets of such similar constitution, mass, and so on, turned out to be so different today. Again, neutron stars are well known now, some of them as pulsars, of which there are many in the sky, but they are not mentioned; nor are quasars nor black holes, but these are not yet understood and they lie on the edge of Einstein's gravitational theory, which the author consciously chose to omit. At the other extreme, the book gives no hint of the complexity of the quark world; we know 15 kinds of quark today, and other fundamental entities are also needed. There is no mention of the weak-decay interactions, the unification of which with electromagnetism to form 'the electroweak interaction' has been such a major theoretical triumph recently. Such topics would perhaps complicate the story without adding much enlightenment, but still they are part of our everyday world. Even the strange particles are being formed around us by the cosmic radiation incident on Earth, although they quickly decay, and this cosmic radiation can be made visible with simple equipment which can be constructed in any home; it is part of our environment. Perhaps the book should have a few appendices, which could take the interested reader further towards the extreme boundaries of present research. I found few errors in the book, but it is stated on p.179 that the highest energy accelerator today, producing proton beams up to 500
$\mathrm{GeV}$, is in operation at the Brookhaven National Laboratory, Long Island; it is actually at the Fermi Laboratory, near Chicago. It also puzzled me that more than half a page should be devoted to the outmoded 'continuous creation' theory of the expanding Universe, definitely excluded by observation for some time past.

In conclusion, I can only say that this book is superb and far ahead of any other global accounting of our Universe, in both the large and the small. We all have friends and relatives who question us often about

\section{Star maps, old and new}

\section{David W. Hughes}

The Sky Explored: Celestial Cartography, 1500-1800. By D. J. Warner. Pp.293. (Alan R. Liss: New York; Theatrum Orbis Terrarum: Amsterdam, 1979.) \$70. True Visual Magnitude Photographic Star Atlas. By C. Papadopoulos. (Pergamon: Oxford and New York.) Three volumes. Vol.1. Southern Stars, $1979 ; £ 100, \$ 225$. Vol.2. Equatorial Stars, 1979; £145, \$325. Vol.3 Northern Stars (by C. Papadopoulos and C. Scovill) publication due June 1980.

SiNCE the dawn of history, mankind has been a fascinated observer of the heavens. The stars were arranged into groups of constellations and names were given to these constellations and also to many of the brighter stars. However, without a good celestial map the night sky simply dissolved into a trackless confusion of points of light. Maps were also a simple means of recording the knowledge gleaned from celestial observations. In the old days the constellations were depicted by artistic, symbolic figures, and many of the early star maps and globes became much more than just scientific aids but were also beautiful works of art in their own right.

The first important extant star catalogue was compiled by Ptolemy in AD 150 and contained 1025 stars grouped into 48 constellations. Ptolemy's work was the cornerstone of mediaeval star catalogues and, for more than 1,400 years, Islamic and European astronomers limited their observations to the stars listed by Ptolemy. The catalogue of Al-Sufi in the tenth century, Ulugh Beg's Alfonsine Tables in the fifteenth century and the Copernicus catalogue in the early sixteenth century were simply revisions of Ptolemy. These catalogues were usually just lists of stellar latitudes, longitudes and magnitudes.

Most astronomers found that a graphic form was more useful than a catalogue, and so maps of the sky were introduced. The first flat star map of any significance was published by Albrecht Durer, the great Nuremberg artist and mathematician, in modern science; this is the book to place in their hands. It shows the physicist at his best, accounting for what he sees by simple order-of-magnitude estimates. The story of Life is less amenable to this approach, and so those chapters are more descriptive. However, as it now is, the book already satisfies a great contemporary need.

R.H. Dalitz is Royal Society Research Professor in the Department of Theoretical Physics at the University of Oxford, UK.

1515. The date reflects both the development of printing and the upsurge in the study of astronomy initiated by such worthies as Copernicus, Regiomontanus and Piccolomini. The era of great celestial cartography probably ends with the publication of John Elert Bode's Uranographia in 1801. Bode used 99 constellations and included 17,240 stars down to eighth magnitude. After Bode the constellation boundaries became more rational, the constellation figures slowly disappeared and star maps lost much of their charm and beauty becoming the dull procession of differing sized dots we know today.

Deborah J. Warner, the curator of the History of Astronomy at the National Museum of History and Technology, Smithsonian Institution, has produced a comprehensive catalogue of all the flat star maps printed in Europe between the fifteenth and early nineteenth centuries. Also included are details of a few globes for comparison. It is a work of considerable scholarship and, as a source of detailed information, will be a much valued reference book for decades to come. The listing is alphabetical and the work of each cartographer is analysed according to the number and size of maps produced, the limiting star magnitude, the co-ordinate system adopted, the date and place of publication, and the non-Ptolemaic constellations introduced. It is intriguing to note how certain constellations come and go. For example Lalande charted Felis, his own cat, and also introduced his telescope as Quadrans Muralis. Thomas Young squeezed the Battery of Volta into the space between the head and hooves of Pegasus. Julius Schiller even attempted to de-paganize the complete zodiac by introducing the twelve apostles in the place of Pisces, Aries, Taurus, Gemini and so on. The Warner catalogue is profusely illustrated in black and white and the beauty of these illustrations is most impressive. Obviously only a few of the maps were coloured and even this would probably have been a laborious task of hand water-colouring. It is hard to fault this book but may I make a suggestion for a new venture. If Deborah Warner produced a large-format book, with some colour prints depicting the best artistic 
representations of each constellation, I'd be first in the queue with my money.

The transition between star maps, the beautiful, and star maps, the functional tool of modern science, is illustrated perfectly by the second book under review. Papadopoulos has produced a large set of maps by photographing the sky through a four-element astrographic objective lens manufactured by Carl Zeiss (Oberkochen). This lens had a diameter of $12.5 \mathrm{~cm}$, a focal length of $62.5 \mathrm{~cm}$ and gave a negative with a usable field of $15^{\circ}$. Each map has been produced from the original negative by line reproduction, a system which prints only black, and has no halftones. This is ideal for stars but gives a very poor reproduction of the appearance of galaxies and nebulae.

The Southern Stars volume contains 120 maps covering the sky region from declination $-30^{\circ}$ to the South Celestial Pole. Each map covers an area of $11^{\circ}$ by $11^{\circ}$ and there is a minimum overlap of at least $1^{\circ}$ between neighbours. The Equatorial Stars volume contains 216 maps and covers the region between $-30^{\circ}$ and $+30^{\circ}$ in declination. A set of transparent co-ordinate overlays are provided, six for Vol. 1 and three for Vol. 2 . The system is converted to 1950.0. Each map measures about $33 \mathrm{~cm}$ by $33 \mathrm{~cm}$. Volume 3, Northern Stars, will be published later this year.

The atlas is designed to show all stars which are brighter than visual magnitude 13.5 and, to quote the author, "the final print produces star images with easily readable differences in their diameters according to their magnitude". The accuracy with which the magnitude can be assessed obviously depends on the accuracy with which the star image diameters have been produced on the map and on the accuracy of measuring these diameters (a star of third magnitude is about $1 \mathrm{~mm}$ across). Superficially each map appears like a white sheet speckled with tiny black dots, surrounded by a black border with right ascension and declination markings on them so that the transparent grid can be positioned. To find your way between maps you are given a rather inadequate, small-scale, chart index. You actually need at least a copy of Antonin Bečvár̆'s Atlas Coeli and preferably a set of Smithsonian Astrophysical Observatory star charts as well. The main advantage of the Papadopoulos atlas is that it goes down to visual magnitudes 13.5 as opposed to the SAO which stops about ninth. The main disadvantage is the price, but assuming you need to go down to $13.5 \mathrm{mag}$ (and I would be very surprised if the number of astronomers who do really need to do this is more than miniscule) $£ 245$ for a collection of 336 Papadopoulos maps - Vols. 1 and 2 - is what you have to pay here. I must confess that I won't, and neither will my university.

David W. Hughes is Lecturer in Astronomy and Physics at University of Sheffield, UK.

\section{Thriving young discipline}

\section{Martin Daly}

Human Sociobiology: A Holistic Approach. By D.G. Freedman. Pp.192. (Free Press/Collier Macmillan: New York and London, 1979.) £8.50. The Genesis Factor. By R.A. Wallace. Pp.256. (William Morrow: New York, 1979.) $\$ 9.95$.

HUMAN sociobiology seems to be a thriving young discipline, if a spate of books and new journals over the past two years is to be taken as evidence. Perhaps much of this activity will eventually be seen to be the cloaking of old ideas in new jargon, but much of it is truly synthetic. Sociobiology is gaining adherents among social scientists because, rather than contesting their established theoretical approaches, it is for the most part metatheoretical to their usual levels of analysis. Theories of sex role socialization, for example, may accurately describe processes by which masculine and feminine behaviour develop, while offering no insight into the cross-cultural consistency of the outcomes. Or a potlatch may be explained as a status competition, while taking it as a given that status is a commodity for which men will find it reasonable to compete. Sociobiological theory attempts to deal with some of the questions that structural, descriptive theories of human action fail to address.

If a "new synthesis" can indeed be said to be emerging, it is not surprising that anthropology, the social science most informed by evolutionary thought, is leading the way. However, sociobiological schools of thought have also surfaced in economics, sociology and even political science. The laggards whose participation is most seriously missed are psychologists.

The grounds for thinking psychology a crucial missing element in a productive synthesis are as follows. Anthropologists and other scientists testing sociobiological hypotheses have been concerned to demonstrate measurable fitness consequences of extant variation in behaviour, and they have achieved some notable successes (see, for example, chapters in Evolutionary Biology and Human Social Behavior: An Anthropological Perspective, edited by N.A. Chagnon and W. Irons; Duxbury Press, 1979). However, a good deal of modern human behaviour is manifestly not fitness-maximizing. Must sociobiology therefore be mute about its origins? Not if appropriate models of the structure of human motives and cognitive processes can be developed. The psychological mechanisms proposed must be anchored simultaneously in sociobiological accounts of their phylogeny and adaptive significance and in predictions of their behavioural outputs, including predictive statements about the kinds of circumstances in which such evolved mechanisms can be expected not to produce adaptive outcomes. This task offers experimental psychologists a gold mine of opportunity for research and for theory development.

Daniel Freedman's Human Sociobiology: A Holistic Approach constitutes an early psychological foray into this rich field, and while the effort is deeply flawed, it nevertheless unearths several nuggets. The book's greatest shortcoming resides in the fact that Freedman ventures out of his depth in population biology. This produces two extremely distracting muddles. One, which surfaces repeatedly, concerns the significance of populational levels of heterozygosity. Freedman imagines that inbreeding is selected against not because of any deleterious effects of homozygosity upon the offspring of consanguineous unions, but because it constitutes "bad evolutionary planning" by reducing populational reservoirs of heterozygosity. He proceeds from this basic misunderstanding to characterize human populations as more or less heterozygous according to their marriage practices, a train of speculation which in Chapter 7 leads to several odd conclusions which leave the author needlessly vulnerable to accusations of racism.

The second major muddle is conceptually related to the first. Freedman has borrowed his University of Chicago colleague Michael Wade's suspicion of

\section{MESOZOIC MAMMALS}

\section{The First Two-Thirds of Mammalian History}

\section{Edited by Jason A. Lillegraven}

Mammals have been an important part of the earth's land fauna for at least 200 million years. This book offers a comprehensive review of what is known about mammalian life during the first twothirds of its history - the Mesozoic era before the extinction of the great reptiles. The book provides biologists and geologists with encyclopedic listings of fossil locations, recognized taxa, and temporal-geographical occurrences of genera along with descriptions of the anatomical features of each group and extensive bibliographies. Interwoven with the summaries of data are valuable interpretive - sometimes admittedly speculative - discussions of the origins of mammals, their patterns of distribution, and their biological, behavioral, and evolutionary relationships during the "dark ages" of mammalian history. Illus. 320 pages $£ 21.00$, cloth $£ 5.75$ paper.

\section{UNIVERSITY OF CALIFORNIA PRESS}

2-4 Brook Street, London W1. 